\title{
ANÁLISE EPIDEMIOLÓGICA DA LEISHMANIOSE TEGUMENTAR AMERICANA
}

\author{
*Daniel Meira Nóbrega de Lima I \\ Maurus Marques de Almeida Holanda "I
}

\section{RESUMO}

A Leishmaniose Tegumentar Americana (LTA)éuma doença infecciosa causada pelo protozoário do gênero Leishmania. Surtos dessa doença são desencadeados pela combinação de fatores biológicos, climáticos e sociais. O desequilíbrio de habitats naturais tem contribuído para o surgimento de novos focos de transmissão da LTA e para a modificação dos padrões epidemiológicos. Os padrões epidemiológicos da LTA, no Nordeste do Brasil, precisam ser melhores descritos. O objetivo desta pesquisa foi traçar o perfil epidemiológico dos casos de Leishmaniose Tegumentar Americana, no Nordeste, no período de 2007 a 2018. Trata-se de um estudo epidemiológico de caráter descritivo e retrospectivo, realizado através dos registros do Sistema de Informação de Agravos de Notificação (SINAN), e dos dados demográficos do Instituto Brasileiro de Geografia e Estatística (IBGE). A partir de 77.030 fichas de notificação, observouse que a maior parte dos casos ocorreu entre pessoas do sexo masculino (62,9\%), de cor de pele parda (70,2\%), de baixa escolaridade (86,5\%) e faixa etária dos $20-59$ anos (56,2\%). A maior parte dos casos que evoluíram para cura (94,5\%) era não-recidivantes (95,4\%), autóctones do município de residência (92,7\%), de forma clínica cutânea $(96,6 \%)$ e foram diagnosticados a partir de exames laboratoriais $(68,6 \%)$. As capitais corresponderam a apenas $1,303 \%$ das notificações e os estados da Bahia e Maranhão são responsáveis por 78,536\% das fichas de notificação. De 2007 a 2018 houve redução de 39,6\% na taxa de incidência de LTA na região Nordeste. A taxa de incidência média dos últimos anos foi de 11,65 casos por 100.000 habitantes.

PALAVRAS-CHAVE: Doenças Endêmicas. Perfil Epidemiológico. Incidência.

Graduando em Medicina. Universidade Federal da Paraíba, UFPB. Departamento de Medicina Interna. CEP: 58051-085, João Pessoa, Paraíba, Brasil. *Autor correspondente: danielmrnobrega@gmail.com ORCID ID: 0000-0001-8384-3895.

Doutor em Medicina. Universidade Federal da Paraíba - UFPB. Departamento de Medicina Interna. CEP: 58051-085, João Pessoa, Paraíba, Brasil. Orcid: 0000-0003-2217-4434. 


\section{INTRODUÇÃO}

\section{A Leishmaniose Tegumentar Americana} (LTA) é uma das patologias infecciosas, não contagiosas, com maior impacto na saúde pública do mundo. Essa zoonose tem como agente etiológico um protozoário do gênero Leishmania e possui uma elevada incidência no Brasil. 1,3 Essa afecção polimórfica se manifesta a partir de apresentações cutâneas, mucosas ou cutaneomucosas. O seu desenvolvimento resulta de uma complexa relação entre fatores imunogenéticos do portador e aspectos do parasita - tais como a defesa imunológica inata e adaptativa, a idade e hábitos do infectado, a quantidade de protozoários inoculados e sua localização e tropismo.4,6

Ademais, a LTA ocasiona deformidades que repercutem na estética, nas relações interpessoais, na autopercepção e no afeto. Ou seja, afeta psicossocialmente, uma vez que esses pacientes passam a ser discriminados socioeconomicamente, verificando uma alta taxa alta de desemprego entre eles. Além disso, o tratamento é dispendioso, de longo período e possui altas taxas de efeitos colaterais. 7,8

O Brasil faz parte do grupo de 10 países em que ocorrem $70-75 \%$ dos casos, sendo caracterizado como uma região endêmica. 6,9 Através da solidificação das ações de vigilância e controle de LTA, observou-se um aumento do número de casos registrados em mais de $1100 \%$, alcançando, em 1995, o seu pico máximo de 35.748 casos. $^{10}$

No século passado, o padrão epidemiológico característico da LTA era o silvestre, ocorrendo, principalmente, em áreas com vegetação primária. Nas últimas décadas, observou-se um aumento de novos padrões, como o ocupacional/lazer e o rural/ periurbano por zonas de colonização. O primeiro está associado a modificação do meio ambiente pelo ser humano e o segundo pelo processo migratório e de ocupação das matas secundárias ou residuais. ${ }^{10}$

A LTA era uma doença típica do meio rural, com uma cadeia de transmissão intrinsecamente associada a processos sociais específicos - como o trabalho-, a ecossistemas, à ocupação espacial e ao uso do solo. Descrever o padrão epidemiológico característico dessa doença, ajudará profissionais de saúde no diagnóstico. A vigilância e a fiscalização em saúde fornecem dados fundamentais para que gestores e profissionais de saúde possam organizar e elaborar programas e intervenções que incidam de forma contundente na cadeia de transmissão, e possibilitem diagnosticar, tratar precocemente e reabilitar esses pacientes. ${ }^{2,11}$ Diante da ampliação das zonas de afecção nos últimos anos, acometendo, inclusive, zonas periurbanas e desmatadas, fazse necessário novos estudos sobre a dinâmica epidemiológica. ${ }^{2}$

Para o Ministério da Saúde, todo caso suspeito por critério clínico-epidemiológico deve ser, preferencialmente, confirmado por método laboratorial - seja por encontro do parasita no parasitológico direto e/ou indireto, por Intradermorreação de Montenegropositiva, ou outro método de diagnóstico positivo. A única exceção baseia-se na impossibilidade de realização de exame complementar que, no caso de lesão mucosa, pode ser considerada como critério de confirmação a presença de cicatriz cutânea. ${ }^{10}$

Dessa forma, o presente estudo tem como objetivo traçar o novo perfil epidemiológico da LTA no Nordeste, no período de 2007 a 2018. Além disso, buscouse averiguar a existência de uma tendência da taxa de incidência na região e calcular as taxas de incidência em cada capital e estado. 


\section{MATERIAL E MÉTODOS}

O presente trabalho é um estudo epidemiológico de caráter descritivo e retrospectivo, comanálise de dadossecundários em saúde por meio das notificações de LTA do SUS. Foram utilizados os registros do Sistema de Informação de Agravos de Notificação (SINAN). Os dados do SINAN foram obtidos por meio do sistema de tabulação TABWIN da plataforma do Departamento de Informática do Sistema Único de Saúde (DATASUS). ${ }^{12}$ A população do estudo foi composta de casos diagnosticados de LTA, no período de 2007 a 2018. No banco de dados, foram excluídos os preenchimentos "em branco" das fichas de notificação.

Os dados demográficos populacionais das capitais, estados e região Nordeste foram adquiridos através do portal do Instituto Brasileiro de Geografia e Estatística (IBGE), que possui as informações demográficas mais acuradas e oficiais, do governo.

Os dados foram processados para o software Statistical Package for the Social Sciences (SPSS), versão 22.0. Foram realizados cálculos defrequência de cada variável referente aos pacientes - dividindo o número de fichas que preencheram a variável (numerador), pelo número total de fichas preenchidas entre as variáveis (denominador). Também, foram feitos os cálculos de proporção e incidência nas capitais, estados e região Nordeste. As taxas de incidência foram calculadas, a cada ano,

\section{RESULTADOS E DISCUSSÃO}

Nas 77.030 fichas de notificação referentes à região Nordeste, no período de 2007 a 2018, observou-se que pacientes com LTA são predominantemente do sexo masculino
VOLUME 18 - NÚMERO 3 - Dez/2020 ISSN ELETRÔNICO 2317-7160

178 utilizando o número de registros (numerador) pela população residente, com base em cem mil habitantes (denominador). Quando o objetivo era calcular a incidência no período, foram somados todos os casos (numerador) e divididos pela média aritmética da população residente no período, multiplicada por doze. ${ }^{10}$

As variáveis analisadas foram subdivididas em três grupos: o primeiro é referente ao perfil sociodemográfico - sexo (masculino e feminino), cor de pele/raça (branca, preta, parda, indígena, amarela); escolaridade (analfabeto, ensino fundamental, ensino médio e ensino superior) e faixa etária (<1a, 1-4 anos, 5-9 anos, 10-14 anos, 15-19 anos, 20-39 anos, 40-59 anos, 60-64 anos, 65-69 anos, 70-79 anos e 80 anos ou mais). O segundo grupo avaliava a forma clínica (cutânea e mucosa), tipo de entrada (caso novo e recidiva), critério de confirmação (clínico-laboratorial e clínicoepidemiológico), autóctone do município de residência (sim e não), evolução do caso (cura, abandono, óbito por LTA, óbito por outra causa, transferência, mudança de diagnóstico), período gestacional $\left(1^{\circ}\right.$ Trimestre, $2^{\circ}$ Trimestre, $3^{\circ}$ Trimestre e idade gestacional ignorada). O terceiro grupo consistia nas capitais (São Luís, Teresina, Fortaleza, Natal, João Pessoa, Recife, Maceió, Aracaju e Salvador), estados (Maranhão, Piauí, Ceará, Rio Grande do Norte, Paraíba, Pernambuco, Alagoas, Sergipe e Bahia), região Nordeste.
(62,9\%), da cor de pele parda (70,2\%) e da faixa etária dos 20-39 anos (34,6\%), seguido por 4059 anos (21,6\%) (Tabela 1). 
TABELA 1: Perfil sociodemográfico dos casos de Leishmaniose Tegumentar Americana (LTA), na região Nordeste, durante o período de 2007 a 2018.

\begin{tabular}{|c|c|c|}
\hline Variáveis & $\mathrm{n}^{*}$ & $\% * *$ \\
\hline \multicolumn{3}{|l|}{ Sexo } \\
\hline Masculino & 48.490 & $62,9 \%$ \\
\hline Feminino & 28.536 & $37,1 \%$ \\
\hline \multicolumn{3}{|l|}{ Cor de pele/raça } \\
\hline Branca & 8.836 & $11,9 \%$ \\
\hline Preta & 11.237 & $15,2 \%$ \\
\hline Parda & 52.018 & $70,2 \%$ \\
\hline Amarela & 829 & $1,1 \%$ \\
\hline Indígena & 1.149 & $1,6 \%$ \\
\hline \multicolumn{3}{|l|}{ Escolaridade } \\
\hline Analfabeto & 7.094 & $12,6 \%$ \\
\hline Ensino fundamental & 41.750 & $73,9 \%$ \\
\hline Ensino médio & 6.866 & $12,2 \%$ \\
\hline Ensino superior & 770 & $1,3 \%$ \\
\hline \multicolumn{3}{|l|}{ Faixa etária } \\
\hline$<1$ ano & 1.304 & $1,7 \%$ \\
\hline $1-4$ anos & 2.833 & $3,7 \%$ \\
\hline $5-3$ anos & 4.836 & $6,3 \%$ \\
\hline 10-14 anos & 6.862 & $8,9 \%$ \\
\hline 15-19 anos & 8.094 & $10,5 \%$ \\
\hline 20-39 anos & 26.663 & $34,6 \%$ \\
\hline 40-59 anos & 16.594 & $21,6 \%$ \\
\hline $60-64$ anos & 2.966 & $3,8 \%$ \\
\hline $65-69$ anos & 2.304 & $3,0 \%$ \\
\hline 70-79 anos & 3.060 & $4,0 \%$ \\
\hline 80 anos ou mais & 1.463 & $1,9 \%$ \\
\hline
\end{tabular}

Em estudo paranaense, os pacientes do sexo masculino eram em $59,2-75 \%$ e a faixa etária média, em relação aos casos encontrados, foi de 56,79 anos, sendo a mais afetada de 31 a 75 anos (62-73,8\%)1317. Contudo, em outros estudos no Brasil foi demonstrada uma maior frequência no sexo feminino $(51,7-64 \%)^{18,19}$ e na faixa dos 20 40 anos. ${ }^{13,15,19}$ Assim, observa-se um alto impacto na atividade econômica, uma vez que afeta, sobretudo, homens na faixa de idade produtiva. No estado do Tocantins, região endêmica da doença, foi observado maior número de casos entre pessoas aposentadas acima de 60 anos $(32,4 \%){ }^{16}$

No Paraná, as pessoas de cor de pele branca foram as mais afetadas, seguidas pelas preta e parda - como observado por Detoni et al.. 17 No entanto, há estudos que sugerem uma maior incidência entre pessoas de pele

$$
\begin{aligned}
& \text { parda }(53-69,1 \%){ }^{16,18} \\
& \text { Apesar de a maioria dos estudos }
\end{aligned}
$$
apontarem que a doença é predominante entre pessoas de baixa escolaridade, ${ }^{14}$ ela foi, também, encontrada entre pessoas de média a alta escolaridade. ${ }^{16}$ Também é observado maior prevalência em zonas rurais. ${ }^{14,19}$ Contudo, estudos recentes demonstraram altas taxas em meio urbano, apresentando até cerca de $50-79 \%$ dos casos. É importante mencionar que a menor incidência na zona urbana está associada a maior população, ou seja, a incidência era de apenas $1 / 3$ da apresentada na zona rural. ${ }^{16,18}$

Neste trabalho, foi verificado que os critérios de confirmação usados foram clínicolaboratorial $(68,6 \%)$ e clínico-epidemiológico $(31,4 \%)$ e a forma clínica predominante foi a cutânea (96,6\%). A maior parte foi de casos novos/não-recidivantes (95,4\%) e autóctones 
do município de residência (92,7\%). Essa maioria dos casos autóctones, corrobora com estudo que demonstrou prevalência de $88 \%$ nessa variável. ${ }^{19}$ Ademais, foi observada uma evolução para cura em $84,5 \%$, ocorrendo óbito em 0,7\% (Tabela 2).

Houve recorrência em 1,7 - 29,2\% dos casos, e $12 \%$ possuíam história familiar. A lesão recidivou em 4-7,4\%, apesar da cicatrização em 75\%.15,17-19 Observou-se que a taxa de abandono do tratamento $(1,8$ - 2\%) tem diminuído, uma vez que em 1991, 49,3\% dos pacientes abandonaram o seu tratamento. ${ }^{16,17}$ Apesar desse resultado ser explicado por uma série de fatores, é necessário evidenciar a consolidação do sistema de fiscalização e vigilância do Brasil para LTA em 1985, bem como a melhoria da assistência desses pacientes na atenção básica e secundária, visto que há um melhor acompanhamento dos casos, sem uma perda relevante de continuidade. Além disso, salienta-se a diminuição na morbimortalidade, como também um enorme impacto ao sistema de saúde, pelo menor número de internações hospitalares e custos de tratamento, dada a resolução de quadros clínicos mais brandos e precoces. ${ }^{10}$

TABELA 2: Distribuição dos casos de Leishmaniose Tegumentar Americana (LTA), do Nordeste, 2007-2018, segundo características clínico-epidemiológicas.

\begin{tabular}{|c|c|c|}
\hline Variáveis & $\mathrm{n}^{*}$ & $\% * *$ \\
\hline \multicolumn{3}{|l|}{ Critério de confirmação } \\
\hline Clínico-laboratorial & 52.810 & $68,6 \%$ \\
\hline Clínico-epidemiológico & 24.220 & $31,4 \%$ \\
\hline \multicolumn{3}{|l|}{ Forma clínica } \\
\hline Cutânea & 74.241 & $96,6 \%$ \\
\hline Mucosa & 2.597 & $3,4 \%$ \\
\hline \multicolumn{3}{|l|}{ Tipo de entrada } \\
\hline Caso novo & 72.293 & $95,4 \%$ \\
\hline Recidiva & 3.510 & $4,6 \%$ \\
\hline \multicolumn{3}{|c|}{ Autóctone do município de residência } \\
\hline $\operatorname{sim}$ & 67.636 & $92,7 \%$ \\
\hline não & 5.323 & $7,3 \%$ \\
\hline \multicolumn{3}{|l|}{ Evolução do caso } \\
\hline Cura & 51.343 & $94,5 \%$ \\
\hline Abandono & 1.004 & $1,9 \%$ \\
\hline Óbito por LTA & 55 & $0,1 \%$ \\
\hline Óbito por outra causa & 321 & $0,6 \%$ \\
\hline Transferência & 541 & $1,0 \%$ \\
\hline Mudança de diagnóstico & 1.050 & $1,9 \%$ \\
\hline \multicolumn{3}{|l|}{ Período gestacional } \\
\hline $1^{\circ}$ Trimestre & 25 & $11,4 \%$ \\
\hline $2^{\circ}$ Trimestre & 77 & $35,0 \%$ \\
\hline $3^{\circ}$ Trimestre & 48 & $21,8 \%$ \\
\hline *** IG ignorada & 70 & $31,8 \%$ \\
\hline
\end{tabular}

Percebeu-se uma tendência de redução da taxa de incidência no Nordeste de 2007 para 2018 (Figura 1). Em 2007, a taxa de incidência era de 12,1 casos/100.00 hab., enquanto que em 2018 essa taxa diminuiu para 7,31 casos/100.000 hab. Assim, ocorreu uma diminuição de 39,6\% da taxa de incidência. Além disso, foi observado um pico do número de casos em 2010, quando ocorreu uma taxa de incidência de 17,66 casos/100.000 hab. (Figura 1).

A região Nordeste (29,4\%), em 
conjunto com a região Norte $(46,1 \%)$, apresenta a maior parte dos casos de LTA do Brasil.20 A incidência no Brasil, entre 1985 e 2005 foi de 18,5 casos/100.000 hab. Na região Norte, foi de 82 a 191 casos/100.000 hab., entre 2003 e 2010. Em pesquisas no Sul do Brasil foi verificada uma incidência de 4,81 casos/100.000 hab., no período entre 1993 a 1998.10,13,14

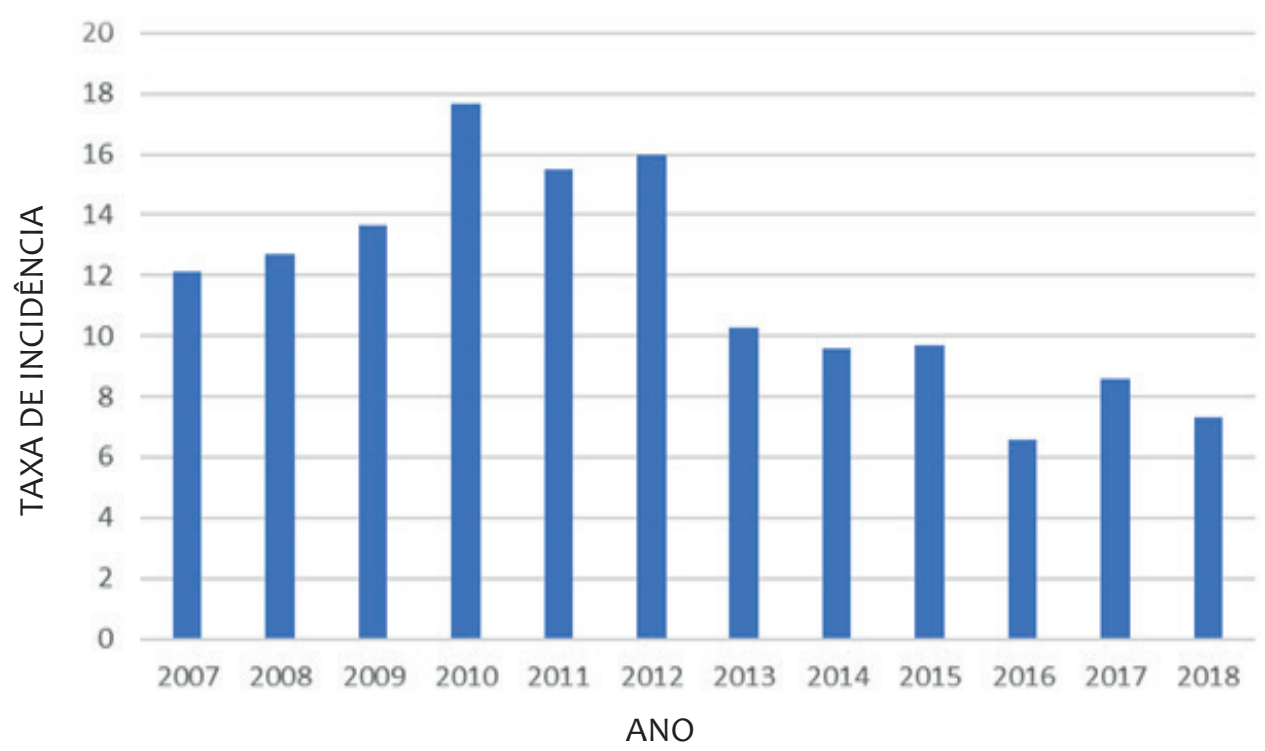

FIGURA 1: Taxa de incidência anual de Leishmaniose Tegumentar Americana (LTA), do Nordeste, durante o período de 2007 a 2018.

Ascapitais commaior taxa deincidência foram Teresina $(3,57)$, São Luís $(0,885)$ e Fortaleza $(0,79)$. Na cidade de Teresina, a região metropolitana é envolta por extensas florestas tropicais, ficando a população mais exposta a reprodução selvagem do parasita nos locais mais periféricos da cidade. ${ }^{21}$

Os estados com maior taxa de incidência foram o Maranhão (29,84), Bahia $(21,3)$ e Ceará $(8,39)$. No caso do Maranhão, há características do bioma amazônico em grande parte do seu território. Na Bahia, a maior incidência pode ser explicada pelo fato de a mesorregião do Sul, onde há uma maior densidade do número de casos, possuir florestas e clima quente/úmido. No estado do Ceará, os dois principais climas são o semiárido e o tropical, sendo a LTA mais prevalente nas regiões tropicais localizadas no norte do estado e próximas ao litoral. ${ }^{10}$ No geral, as capitais tiveram menores taxas do que seus estados - possuindo apenas $1,303 \%$ da quantidade de casos totais, uma vez que há uma intensa urbanização nesses polos. Os estados da Bahia e Maranhão têm aproximadamente $78,536 \%$ do número de casos do Nordeste (Tabela 3).

Apesar do grande número de casos de LTA descritos nesta pesquisa, há uma alta taxa de subnotificação dessa doença, como ocorre com outras patologias. Além disso, é importante salientar que há uma grande quantidade de preenchimentos "em branco" nas fichas de notificação, o que diminui a qualidade das informações sobre esses casos.

A partir das observações desta 
pesquisa, trabalhos futuros devem demonstrar e caracterizar fatores de risco para LTA, através de estudos coorte. Além disso, é necessário incorporar nas fichas de notificação a classificação dos casos em zona urbana, periurbana e rural, bem como diferenciar as formas cutaneomucosas, das cutâneo e mucosas.

TABELA 3: Taxas de incidência média (I) de Leishmaniose Tegumentar Americana (LTA) em capitais e estados da região Nordeste, durante o período de 2007 a 2018.

\begin{tabular}{cccc}
\hline Variáveis & $\mathbf{n}^{*}$ & $\% * *$ & $\mathbf{I}$ \\
\hline \hline São Luís & 109 & $0,141 \%$ & 0,885 \\
Maranhão & 23.554 & $30,578 \%$ & 29,84 \\
\hline \hline Teresina & 352 & $0,457 \%$ & 3,57 \\
Piauí & 1.403 & $1,821 \%$ & 3,71 \\
\hline \hline Fortaleza & 241 & $0,313 \%$ & 0,79 \\
Ceará & 8.687 & $11,277 \%$ & 8,39 \\
\hline Natal & 12 & $0,016 \%$ & 0,12 \\
Rio Grande do Norte & 218 & $0,283 \%$ & 0,56 \\
\hline João Pessoa & 20 & $0,026 \%$ & 0,23 \\
Paraíba & 733 & $0,952 \%$ & 1,60 \\
\hline Recife & 80 & $0,104 \%$ & 0,42 \\
Pernambuco & 4.603 & $5,975 \%$ & 4,27 \\
\hline \hline Maceió & 27 & $0,035 \%$ & 0,24 \\
Alagoas & 800 & $1,039 \%$ & 2,10 \\
\hline Aracaju & 15 & $0,019 \%$ & 0,21 \\
Sergipe & 90 & $0,117 \%$ & 0,36 \\
\hline \hline Salvador & 148 & $0,192 \%$ & 0,43 \\
Bahia & 36.942 & $47,958 \%$ & 21,3 \\
\hline \hline Todas as capitais & 1.004 & $1,303 \%$ & 0,70 \\
\hline Todas os estados & 77.030 & $100 \%$ & 11,65 \\
\hline & & $*$ n: número de casos de LTA \\
\hline \hline & & & \\
\hline \hline
\end{tabular}

\section{CONCLUSÃO}

A Leishmaniose Tegumentar Americana é uma patologia que afeta principalmente o sexo masculino, na faixa etária produtiva dos 30 aos 75 anos, de baixa escolaridade e cor de pele parda. Bem como, há uma maior incidência no meio rural.

O número de casos recorrentes e de abandono tem diminuído. Visto que, o sistema de vigilância e fiscalização tem melhorado, assim como a assistência primária e secundária, possibilitando o diagnóstico, acompanhamento e tratamento de forma efetiva.

Os profissionais de saúde utilizaram, na maioria dos casos, exames complementares para auxiliar no diagnóstico desses pacientes, sendo o teste intradérmico o exame mais empregado.

A taxa de incidência no Nordeste diminuiu durante o período de 2007 a 2018, sendo observado uma redução de quase $40 \%$. Os estados do Maranhão e Bahia realizaram a grande maioria das notificações, sendo as regiões endêmicas com mais prevalência dessa doença. Por fim, as capitais têm baixa incidência dessa doença, sendo mais comum em regiões interioranas. 


\title{
EPIDEMIOLOGICAL ANALYSIS OF AMERICANTEGUMENTARY LEISHMANIASIS
}

\begin{abstract}
American Cutaneous Leishmaniasis (ACL) is an infectious disease caused by the protozoan of the genus Leishmania. Outbreaks of this disease are triggered by a combination of biological, climatic and social factors. The imbalance of natural habitats has contributed to the emergence of new foci of transmission of ACL and to the modification of epidemiological patterns. The epidemiological patterns of $A C L$, in Northeastern Brazil, need to be better described. The objective of this research was to trace the epidemiological profile of cases of American cutaneous leishmaniasis in the Northeast, in the period from 2007 to 2018. This is a descriptive and retrospective epidemiological study carried out through the records of the Notifiable Diseases Information System (Sinan), and demographic data from the Brazilian Institute of Geography and Statistics (IBGE). From 77,030 notification forms, it was observed that the majority of cases occurred among people of the male sex (62.9\%), of brown skin color (70.2\%), with low education (86.5\%) and the 20-59 age group (56.2\%). Most of the cases that evolved to cure (94.5\%) were non-recurring (95.4\%), autochthonous in the municipality of residence $(92.7 \%)$, in a clinical cutaneous form (96.6\%) and were diagnosed from laboratory tests (68.6\%). Capitals accounted for only $1.303 \%$ of notifications and the states of Bahia and Maranhão are responsible for $78.536 \%$ of notification forms. From 2007 to 2018 there was a 39.6\% reduction in the incidence rate of ACL in the Northeast region. The average incidence rate in recent years was 11.65 cases per 100,000 inhabitants.
\end{abstract}

KEYWORDS: Endemic Diseases. Health Profile. Incidence.

\section{REFERÊNCIAS}

1. Mouttaki T, Yuste MM, Espinisa GM, Chiheb S, Fellah H, Sanchez JM, et al. Molecular diagnosis of cutaneous leishmaniasis and identification of the causative Leishmania species in Morocco by using three PCR-based assays. Parasit Vectors. 2014; 7(420): 1-9.

2. Abuzaid AA, Abdoon AM, Aldahan MA, Alzahrani AG, Alhakeem R, Asiri AM, et al. Cutaneous leishmaniasis in Saudi Arabia: A comprehensive overview. Vector Borne Zoonotic Dis. 2017; 17(10): 673-84.

3. Maleki M, Yousefi M, Bazzaz SM, Tabassi SA, Rakhshandeh H, Hamedi SS, et al. An overview of skin lesions adapted to cutaneous leishmaniasis in persian medicine. Electron Physician. 2017;
$9(11): 5854-62$.

4. Maspi N, Abdoli A, Ghaffarifar F. Pro-and anti-inflammatory cytokines in cutaneous leishmaniasis: a Review. Pathog Glob Health. 2016; 110(6): 247-60.

5. Meireles CB, Maia LC, Soares CC, Teodoro IP, Gadelha MS, Silva CG, Lima MA. Atypical presentations of cutaneous leishmaniasis: A systematic review. Acta Trop. 2017; 172: 240-54.

6. Gabriel A, Bolas A, Marques J, Gonçalves P, Ruas P, Guerreiro T, Gomes G. Cutaneous leishmaniasis: The complexity of host's effective imune response against a polymorphic parasitic disease. J Immunol Res. 2019; e2603730. 
7. Handler MZ, Patel PA, Kapila R, Qubati YA, Schwartz RA. Cutaneous and mucocutaneous leishmaniasis: Differential diagnosis, diagnosis, histopathology, and management. J Am Acad Dermatol. 2015; 73(6): 911-26.

8. Aronson NM, Joya CA. Cutaneous leishmaniasis: Updates in diagnosis and manegement. Infect Dis Clin of North Am. 2019; 33(1): 101-17.

9. Burza S, Croft SL, Boelaert M. Leishmaniasis. Lancet. 2018; 392(10151): 951-70.

10. Ministério da Saúde (BR). Secretaria de Vigilância em Saúde. Departamento de Vigilância das Doenças Transmissíveis. Manual de vigilância de leishmaniose tegumentar. Brasília, 2017. Disponívelem:http://bvsms.saude.gov.br/bvs/ publicacoes/manual_vigilancia_leishmaniose_ tegumentar.pdf. Acesso em: 27 jul 2019.

11. Laboudi $M$, Sahibi $H$, Elabandouni $M$, Nhammi $\mathrm{H}$, Hamou S, Sadak A. A review of cutaneous leishmaniasis in Morocco: A vertical analysisto determine appropriate interventions for control and prevention. Acta Trop. 2018; 187: 275-83.

12. Ministério da Saúde (BR). Departamento de Informática do Sistema Único de Saúde. Brasília, 2019. Disponível em: http://www2.datasus.gov. br/. Acesso em: 24 jun 2019.

13. Castro EA, Soccol VT, Membrive N, Luz E. Estudo de características epidemiológicas e clínicas de 332 casos de leishmaniose tegumentar notificados na região norte do Estado do Paraná de 1993 a 1998. Rev Soc Bras Med Trop. 2002; 35: 445-52.

14. Guzmán HO, Martins AC, Mantovani SA, Brana AM, Delfino BM, Pereira TM, et al. Características epidemiológicas da leishmaniose tegumentar americana na fronteira amazônica: estudo retrospectivo em Assis Brasil. Acre. 2013; 42(2): 187-200.
15. Rocha TJ, Santana EP, Barbosa AC, Calheiros CM. Aspectos epidemiológicos de casos humanos confirmados de leishmaniose tegumentar americana no estado de Alagoas, Brasil. Rev Pan-Amaz Saude. 2015; 6: 49-54.

16. Gosch CS, Marques CP, Resende BS, Souza JS, Rocha RA, Lopes DS, et al. American tegumentary leishmaniasis: epidemiological and molecular characterization of prevalent Leishmania species in the State of Tocatins, Brazil, 2011-2015. Rev Inst Med Trop São Paulo. 2017; 59: e91.

17. Detoni MB, Lima DM, Silva TP, Machado LF, Pellissier FT, Costa IN, et al. Temporal and spatial distribution of American tegumentary leishmaniasis in north Paraná: 2010-2015. Rev Soc Bras Med Trop. 2019; 52: e20180119.

18. Xavier KD, Mendes FC, Barbosa LA. Leishmaniose tegumentar americana: estudo clínico-epidemiológico. Rev Unincor. 2016; 14(2): 120-22.

19. Santos JC, Binow AM, Santos S. Estudo de incidência de casos de leishmaniose tegumentar americana no município de Espigão do Oeste RO. Revesc. 2016; 2(1): 15-28.

20. Alcântara LR, Demarchi IG, Aristides SM. Evolução dos casos de leishmaniose tegumentar americana relacionados no estado do Paraná, Brasil. Rev Inst Med Trop São Paulo. 2016; 58(67): 1-7.

21. Wernecke GL, Pereira TJ, Farias GC, Silva FO, Chaves FC, Gouvêa MV, et al. Avaliação da efetividade das estratégias de controle da leishmaniose visceral na cidade de Teresina, Estado do Piauí, Brasil: resultados do inquérito inicial - 2004. Epidemiol. Serv. Saúde. 2008; 17(2): 87-96. 